\title{
Modeling of Power Consumption of the Mining Equipment Using "The Probabilistic Automata Method"
}

\author{
Alla Zakharova ${ }^{1}$, Veniamin Kashirskikh ${ }^{1 *}$, Irina Lobur $^{1}$, Nadezhda Shauleva ${ }^{1}$ and Valeriy \\ Borovtsov ${ }^{1}$ \\ ${ }^{1}$ T.F. Gorbachev Kuzbass State Technical University, 28, Vesennaya st., 650000, Kemerovo, Russia
}

\begin{abstract}
The method of power consumption forecasting based on hierarchy of the objects forming system is considered. One of perspective methods of numerical modeling of casual processes, i.e. direct generation of the studied process realization by means of the Monte Carlo method is involved in the solution of this task. The mining equipment belongs to systems with final number of states, and the separate realization of their evolution process is enabled by method of "probabilistic automata method" which generates transitions between conditions of the system by some rules set in advance. The parameters which form the basis for drawing up a matrix of probabilities of transitions when modeling a running cycle of the shearer using this method are determined on the basis of statistical data. The regularities of power consumption by separate units, subsystems and the enterprise in general established by means of the offered model can be applied to the solution of a problem of increase in efficiency of the electric power use both at a design stage, and to conditions of normal operation of the enterprise.
\end{abstract}

\section{Introduction}

The power economy of the coal-mining enterprise is a difficult system. Its functioning is defined by the nature of interrelations between its elements, and also external and internal factors a part of which has casual character. At the same time, one of the main tasks arising is forecasting of electric energy costs for production of one product. To solve this task, it is required to know statistical characteristics of electric energy consumed by both the mining equipment (ME), and the received final product [1-6].

To forecast electricity consumption, it is necessary to solve an important question, namely, the record of casual impacts on the units of the technological chain as casual impacts on separate units form dispersions of system characteristics in advance up a hierarchy of the objects forming system (the coal enterprise).

\footnotetext{
${ }^{*}$ Corresponding author: kvg.ea@kuzstu.ru
} 


\section{Theoretical basics}

The expected scheme of modeling of power consumption by the coal enterprise can be constructed by the principle of the "ascending stream of models" [7] based on the hierarchy of the objects forming system the essence of which is as follows. At the beginning, the behavior of objects of the lowermost level of hierarchy "units" is modeled (for example, some types of mining equipment: shearers, conveyors, belt elevators, pumps of the main mine drainage, etc.) at the set input characteristics of casual influences. The lower level of objects is characterized by the fact that its elements can't be separated into smaller components. Objects of the lowermost level - "units" - experience casual influences.

Output characteristics of "units" are input characteristics for conditions of the following level of hierarchy - "subsystems" (the set of mining equipment united by a certain feature, for example the mining equipment of certain mining districts).

The input parameters for modeling of objects of the following hierarchical level are received by modeling output characteristics of all objects of the hierarchical level second from below on the input parameters received on the previous step. The described process of advance up hierarchy continues until behavior of the "system" (the coal enterprise), the object of the highest hierarchical level, is simulated.

Creation of the expected scheme described above requires establishment of regularities of power expense formation in the form of the mathematical models considering influence of casual influences that demands attraction of the mathematical theory of casual processes describing transitions of system between its states under the influence of casual influences $[8,9]$. For creation of mathematical model of a casual process it is necessary to define the number of the system's conditions and probability of system's transitions between its states. Further it is possible to simulate trajectories of casual process, the sequence of system's transitions between its states.

One of the most perspective methods of numerical modeling of casual processes is the method of direct generation of the studied process by means of the Monte Carlo method [10-15] based on generation of random numbers. For systems with final number of states to which ME belongs the separate realization of their process evolution can be carried out by one of perspective methods of direct modeling of dynamics of stochastic systems - a method of "probabilistic automata" [16].

"The probabilistic automata" is the system with final number of states $E_{1}, E_{2}, E_{3} \ldots, E_{B 1}$ the transitions between which are described by probabilities of transition $P_{i j}$. "The probabilistic automata" generates casual transitions by means of the rule defined in advance and based on generation of random numbers. This rule also defines the structure of "the probabilistic automata". Modeling of dynamics of the system by means of "the probabilistic automata" is realization of the casual process described by Chapman-Kolmogorov's equation for the total probability of transition for any number of actions.

$$
\operatorname{Pr}\left\{E_{i} \rightarrow E_{j} \text { actions from } \mathrm{k} \text { to } \mathrm{k}+\mathrm{m}\right\}=\sum_{\left\{n_{1}\right\}} P_{i n_{1}}(k) P_{n_{1} n_{2}}(k+1) \ldots P_{n_{m-1}}(k+m) .
$$

With set the initial condition of the system, the sensor of random numbers defines into what admissible states the system has to pass, and the system is transferred to the following state. On the following steps of the considered temporary interval, the same procedure of determination of probability of transition and transfer of the system to the corresponding state is carried out.

As a result of the described process of casual transitions during all considered time interval, concrete realization of casual process turns out - the casual sequence of states in which the system stayed in simulated process of evolution

$$
s_{0}, s_{1}, s_{2}, s_{3} \ldots, s_{N},
$$

where $s_{k}$ - number of the state in which the system was on step with number $k$. 
Based on the received sequence of states, temporary dependence of any measured size connected with evolution of system can be constructed.

After the task of the system conditions, the matrix of probabilities of transitions is defined

$$
P_{i j}(d t)=\left\{\begin{array}{c}
1-\lambda_{i}(t) d t, i=j ; \\
a_{i j}(t) d t, i \neq j,
\end{array}\right.
$$

where value $\lambda_{i}(t) d t$ makes sense to probability of exit of the system from condition $E_{i}$ for time interval $(t, t+d t)$. The parameters characterizing the conditions of the system and the matrix of probabilities of transitions form a set of input characteristics of the model. Further calculation of output parameters and the analysis of results is made.

\section{Application}

For modeling the work of the coal-mining enterprise unit with use of the considered method as an example the operating cycle of shearer KGS-445 has been chosen as the shearer is the main technological machine performing loading to all units and the mine in general.

Now extensive material about determination of average duration of starting-up $T_{\text {inc }}$, duration of operating time tp and duration of operating cycle of shearer tц during shift [7]. Duration of starting-up $\mathrm{T}_{\text {inc }}$ of shearers with the modes of frequent start-up is determined by the formula

$$
\mathrm{T}_{i n c}=\frac{\left(n t_{s}+t_{w}\right)}{t_{c}},
$$

where $n$ - number of shearer motors; $t_{s}$ - duration of the motor starting; $t_{w}$ - work duration under loading; $t_{c}=n t_{s}+t_{w}+t_{0}$ cycle duration; $t_{0}-$ pause time. Start-up duration for faces with daily output to $1200 \mathrm{t}$ makes $0.5-0.68$; with output of $2000-2500 \mathrm{t}-0.7-0.81$; with output above $2500 \mathrm{t}-0.85$.

Distribution of the loading levels during an operating cycle is shown in Fig. 1. During time $t_{\min }$ the first motor (lower drum) starts and runs idling then during time $t_{\text {min.av }}$ the motor of the top drum starts and runs idling. Further, after short time of load increase (it makes 3 $-5 \mathrm{~s}$. therefore it is neglected), the shearer runs with some loading which histogram of distribution is presented on Fig. 2. It is convenient to use this histogram for modeling.

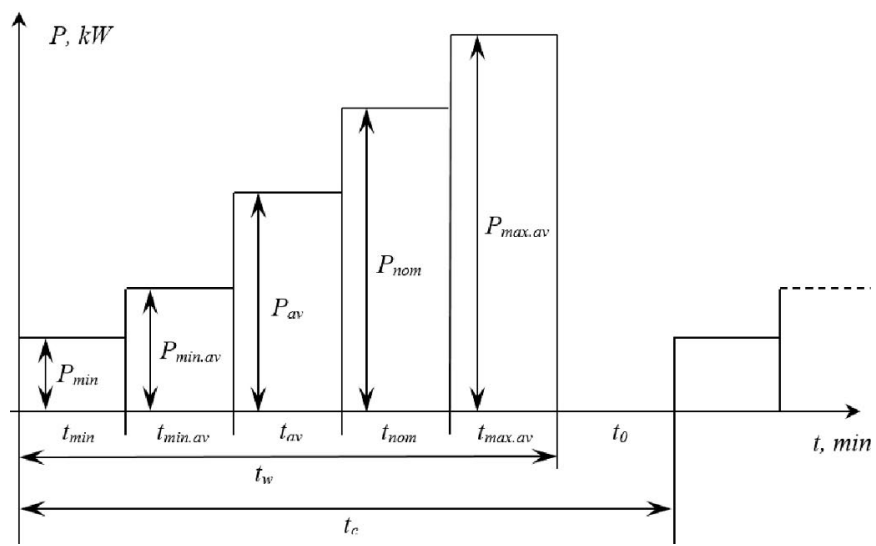

Fig. 1. Distribution of shearer capacity levels during operating cycle 
During a production run which lasts from 25 to $80 \mathrm{~min}$. it is possible to identify five levels of shearer loading: minimum $P_{\min }$; minimum average $P_{\text {min.av }}$; average $P_{a v}$; nominal $P_{\text {nom }}$; the maximum average $P_{\text {max.av }}$.

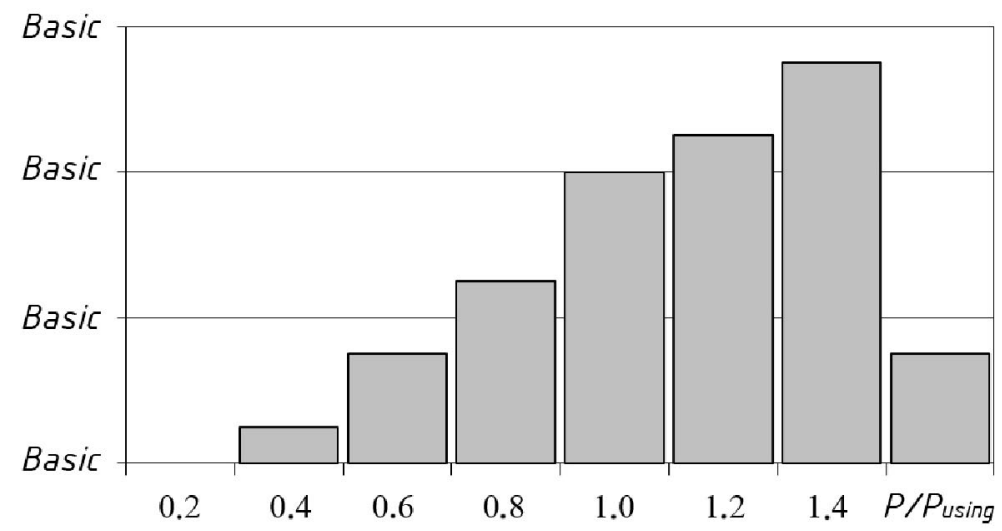

Fig. 2. Histogram of loading distribution of the shearer

The analysis of time observations of production at faces has shown:

- duration of the shearer stops for the intra face and extra face technical reasons has gamma distribution with density

$$
f(t)=\frac{\lambda^{\eta}}{\Gamma(\eta)} t^{\eta-1} e^{-\lambda t}
$$

where, $\lambda, \eta$ - parameters of elementary distributions; $\Gamma(\eta)$ - gamma function;

- duration of stops for the extra face organizational reasons also has gamma distribution, and on intra face ones - exponential with density

$$
f(t)=\lambda e^{-\lambda t}
$$

- duration of stops because of mining-and-geological violations is described by exponential distribution;

- the probability of all types of stops is distributed by Poisson's law:

$$
P(m)=\frac{\lambda^{m}}{m !} e^{-\lambda}
$$

Operating time of shearers under loading has the exponential law of distribution, and duration of the cycle is described by Weybull-Gnedenko's distribution:

$$
f\left(t_{c}\right)=\frac{b}{a}\left(\frac{t_{c}}{a}\right)^{b-1} \exp \left[-\left(\frac{t_{c}}{a}\right)^{b}\right]
$$

where $a, b$ - distribution parameters. Observations have shown that the output per face of $1500 \mathrm{t} /$ day, the cycle duration with confidential probability $p=0.9$ is in the range of 40 to 50 min., and at the output up to $2500 \mathrm{t} /$ day - up to $78 \mathrm{~min}$. The faces working with output of 1.0 million tons/year are characterized by long cycle up to $135-180 \mathrm{~min}$. Having the presented statistical data, it is possible to calculate probabilities of continuous work and a stop of the shearer, and also probability of continuous work in minutes will make no more $t_{w}$ and duration of stops won't exceed $t_{o}$. 
In the considered model during a running cycle of the shearer KGS-345 six temporary periods described in Table 1 are allocated, and amount of the consumed electric power is submitted through the total rated power of cutting motors $P_{\text {nom.m }}$ and rated power of the drive motor $P_{\text {nom.d. }}$. To estimate probabilities of transition between the considered conditions of the shearer we will use results of researches [7] reporting us that the average duration of stops for various reasons makes $8 \div 28 \mathrm{~min}$, and probabilities of shearer stops lie in the range of $0,04-0,52$.

Table 1. Designation of a shearer condition

\begin{tabular}{|c|c|c|c|}
\hline Period & Description & Power consumption & Designation of condition \\
\hline$t_{0}$ & Motors are switched off & Absent & E0 \\
\hline$t_{\text {min }}$ & $\begin{array}{c}\text { Motors of the lower drum } \\
\text { idle }\end{array}$ & $P_{\text {min }}=(0,12 \div 0,16) \cdot P_{\text {nom.m }}$ & $\mathrm{E} 2$ \\
\hline$t_{\text {min.av }}$ & $\begin{array}{c}\text { Motors of the lower and } \\
\text { top drum idle }\end{array}$ & $P_{\text {min.av }}=(0,26 \div 0,35) \cdot P_{\text {nom.m }}$ & $\mathrm{E} 3$ \\
\hline$t_{a v}$ & $\begin{array}{c}\text { The shearer works with } \\
\text { average output }\end{array}$ & $P_{a v}=(0,7 \div 0,8) \cdot P_{\text {nom.m }}+0,8 \cdot P_{\text {nom.d }}$ & $\mathrm{E} 4$ \\
\hline$t_{\text {nom }}$ & $\begin{array}{c}\text { The combine works with } \\
\text { a rated load }\end{array}$ & $P_{\text {nom }}=P_{\text {nom.m }}+P_{\text {nom.d }}$ & $\mathrm{E} 5$ \\
\hline$t_{\text {max.av }}$ & $\begin{array}{c}\text { The shearer works with } \\
\text { the maximum load }\end{array}$ & $P_{\text {max.av }}=(1,06 \div 1,36) \cdot P_{\text {nom.m }}+P_{\text {nom.d }}$ & \\
\hline
\end{tabular}

\section{Conclusion}

The parameters of the running cycle shown in the form of the graph in Fig. 3 have been determined on the estimates of probabilities of transition and calculation of states parameters. Tops of the graph correspond to conditions of the system, arrows are the transitions from one state to another (where $a-$ transition speed from one state to another).

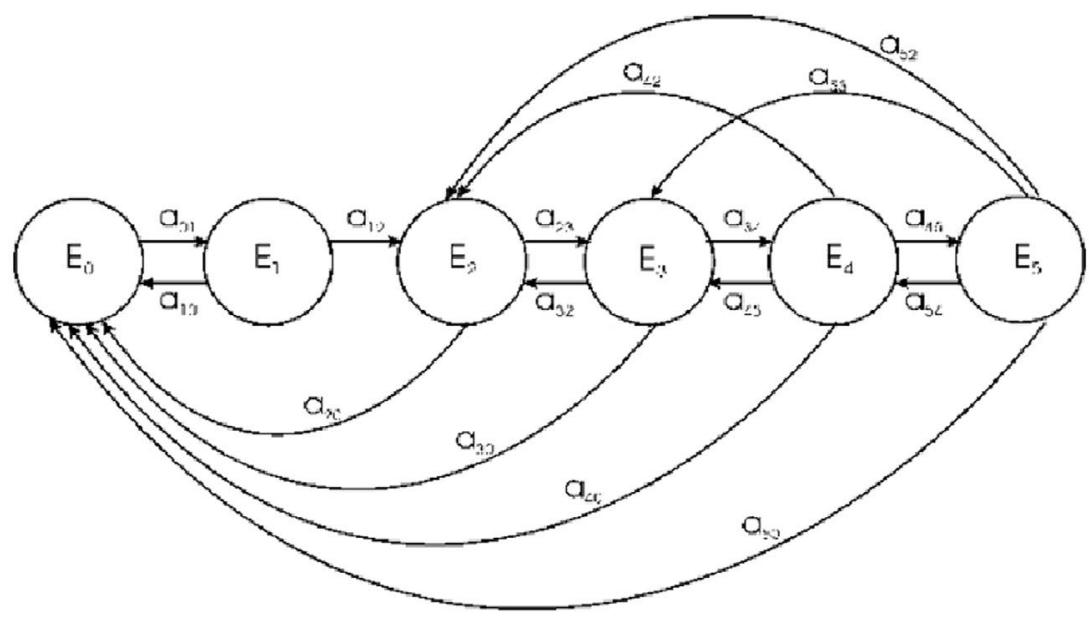

Figure 3. State graph of the "shearer" system 
These data are the basis for drawing up the matrix of probabilities of transitions (1) corresponding to the graph by means of which input parameters for modeling of a running cycle of the shearer KGS-445 can be created.

Established by means of the offered model, the regularities of power consumption of certain units, subsystems and the enterprise in general can be applied to the solution of improving efficiency of the electric power use both at a design stage, and in the conditions of normal operation of the enterprise by optimizing the ME choice.

\section{References}

1. D. W. Bunn, E. D. Farmer, Comparative models for electrical load forecasting (Wiley, New York, 1985)

2. D. W. Bunn, E. R. Larsen, Systems Modelling for Energy Policy (Wiley, New York, 1997)

3. M. Munasinghe, P. Meier, Energy Policy Analysis and Modelling (Cambridge University Press, Cambridge, 1993)

4. J. Taylor, L. de Menezes, P. McSharry, International Journal of Forecasting, 22, 16 (2006)

5. R. Weron, Modeling and Forecasting Electricity Loads and Prices: A Statistical Approach (Wiley, Chichester, 2006)

6. J. Taylor, International Journal of Forecasting, 24, 645 (2008)

7. A. G. Zakharova, I. A. Lobur, N. M. Shauleva, V. A. Borovtsov, Coal in the 21st Century: Mining, Processing and Safety, 198 (Atlantis Press, 2016)

8. N. R. Draper, H. Smith. Applied Regression Analysis (John Wiley \& Sons, New York, 1998)

9. K. Dougherty, Introduction to econometrics (Oxford university press, New York Oxford, 2001)

10. J. C. Bogdanoff and F.Kozin. Probabilistic Models of Cumulative Damage (John Wiley \& Sons, New York, 1985)

11. D. W. Heerman, Computer Simulations Methods in Theoretical Physics (SpringerVerlag, Berlin, 1986)

12. L. Yu. Barash, L. N. Shchur, Computer Physics Communications. 185, 1343 (2014)

13. L. Yu. Barash, L. N. Shchur, Computer Physics Communications. 184, 2367 (2013)

14. B. Stickler, E. Schachinger, Basic concepts in computational physics (Springer International Publishing, Switzerland, 2016)

15. M. Newman, Computational physics (CreateSpace Independent Publishing Platform, USA, 2012)

16. H. Gould, J. Tobochnik, W. Christian, An Introduction to Computer Simulation Methods. Applications to Physical System (Addison-Wesley, 2006) 